\title{
Successful endoscopic closure of a refractory postoperative rectovaginal fistula
}

\section{다)(요 $\odot$}

\author{
Authors \\ Institution \\ Department of Internal Medicine, Komatsu Municipal \\ Hospital, Ishikawa, Japan \\ submitted 4.1.2019 \\ accepted after revision 12.3.2019 \\ Bibliography \\ DOI https://doi.org/10.1055/a-0895-5573 | \\ Endoscopy International Open 2019; 07: E796-E799 \\ (c) Georg Thieme Verlag KG Stuttgart · New York \\ elSSN 2196-9736 \\ Corresponding author \\ Yutaka Matano, Ho-60 Mukaimotoori-matchi, Komatsu \\ 923-8560, Japan \\ Fax: +81-761-21-7155 \\ matano@hosp.komatsu.ishikawa.jp
}

Yutaka Matano, Megumi Zianne, Hitoshi Omura, Noriaki Hayashi, Kazuhiro Miwa

\begin{abstract}
Background and study aims A 71-year old female who received low anterior resection for rectal cancer visited Komatsu Municipal Hospital with complaints of passing feces from the vagina. Endoscopic examination revealed a postsurgical rectovaginal fistula. Medical approaches, including nonoperative management, initial endoscopic closure, transvaginal and endorectal surgery, and fecal diversion, were unsuccessful. Therefore, the patient underwent endoscopy with a pre-procedural endoscopic creation of mucosal pin holes around the fistula opening and clip insertion into the rectal wall in combination with electrocautery and clip closure. This novel approach was effective in achieving permanent closure of the fistula in a single procedure. Most rectovaginal fistulas are surgically managed, however, surgery may be more difficult, invasive, and unsatisfactory for refractory fistulas. Although endoscopic treatment with over-the-scope clips has been increasingly used as a less invasive approach for gastrointestinal fistulas with favorable results, it is not as effective for refractory rectovaginal fistulas. As a minimally invasive surgical procedure, this approach might be effective in small rectovaginal fistulas, particularly refractory ones.
\end{abstract}

\section{Introduction}

Rectovaginal fistula (RVF) is a chronic gastrointestinal fistula defined as an epithelium-lined abnormal communication between the rectum and the vagina. The most common causes of RVFs are obstetric trauma, particularly during the postpartum period, local infection, and rectal surgery. Low anterior rectal resection is the most common cause of RVF secondary to rectal resection, with a frequency of up to $10 \%$ [1]. RVFs are usually managed both medically and surgically; however, there are no standard treatment approaches for cases that fail surgical treatment and are, thus, refractory.

Although a recent study reported on a patient with RVF who was satisfactorily managed with through-the-scope clips (TTSC) [2], there are no reports for endoscopic treatment of refractory RVFs. Recently, over-the-scope clips (OTSC) have been applied for closure of gastrointestinal defects. However, the rate of successful closure of fistulae is less than $50 \%$ and is af- fected by presence of induration or fibrosis [3]. Thus, there are no available treatments for refractory RVF. Here we present the case of a patient with refractory RVF after low anterior resection of rectal cancer who was successfully treated with endoscopic surgery in combination with pre-procedural creation of small holes around the fistula electrically and clip insertion into rectal wall.

\section{Case report}

A 71-year-old female was admitted to our hospital with anal bleeding in May 2013. She had a medical history of acute myocardial infarction in November 2009, hypertension, and psoriasis vulgaris. She had an appendectomy at age 15 years. She underwent colonoscopy, which revealed a sessile polyp, approximately $2 \mathrm{~cm}$ in diameter, at the anterior wall of the upper rectum. Biopsy revealed presence of a well- to moderately-differ- 
entiated tubular adenocarcinoma. Laparoscopy-assisted low anterior rectal resection was performed in August 2013.

On Day 14 after surgery, the patient noticed passage of feces from her vagina. Barium enema showed a leak of contrast medium to the vagina. To achieve decompression in the rectal cavity, an indwelling drain was inserted into the woman's rectum, and she did not receive anything orally, except for small volumes of water. One week after drain placement, she underwent sigmoidoscopy, which revealed a small fistula opening at the anastomotic site of the anterior rectal wall. Therefore, endoscopy was performed. First, electrocautery via argon plasma coagulation (APC) was used to burn the epithelium around the fistula opening, followed by application of metal hemoclips with intent to close the opening. However, the rectal wall around the fistula opening was very tight due to fibrosis for an efficient penetration of hemoclips, and the fistula could not be closed. Therefore, the patient underwent transvaginal surgical closure the following day and endorectal and concurrent repeated transvaginal closure of the fistula and ileostomy 12 days after the first transvaginal surgery. However, she continued to pass mucus and a small amount of fecal discharge through her vagina for 4 months. Endoscopic examination in January 2014 revealed a tiny slit-like opening in the anastomotic stoma ( $\triangleright$ Fig. 1 ). The patient was scheduled to undergo a novel endoscopic procedure after she had provided written informed consent. First, four mucosal pin holes were electrically created on both sides of the fistula opening using a needle knife. Next, similar to the previous endoscopic procedure, the epithelium around the opening was electrocauterized via APC. Clip prongs were inserted in the incisional holes across the opening, and the opening was then carefully closed. The same procedure was performed for all incisional holes, and the opening was completely closed ( $\boldsymbol{F}$ Fig. $\mathbf{2 a - d}$ ). After treatment, the patient did not report any vaginal discharge. After 6 months, her ileostomy was subsequently closed and her recovery was uneventful. At 13 months after successful closure of RVF, the patient was free of colonic symptoms, and fistula healing was endoscopically confirmed ( $\triangleright$ Fig. 3 ).

\section{Discussion}

Although RVFs are usually managed both medically and surgically, the surgical approach is preferable. Management of RVFs depends on their size, location, and cause; anal sphincter function; and the patient's overall health status. RVFs can be classified as "low," in which the tract is present between distal anal canal (dentate line or below) and the inside of the vaginal fourchette; "high," in which the tract connects the upper vagina (at the level of the cervix) with the rectum; and "middle," in which the tract lies somewhere in between. In this case, the patient had a high and small RVF, for which an abdominal approach to repair is recommended [4]. Although primary surgical repair of RVFs is associated with a success rate of $70 \%$ to $97 \%$, outcomes reportedly were worse in patients undergoing RVF repair after one or more prior failed repairs [5]. In case presented here, nonoperative management, an initial endoscopic procedure, endorectal and transvaginal closure, and ileostomy were unsuc-

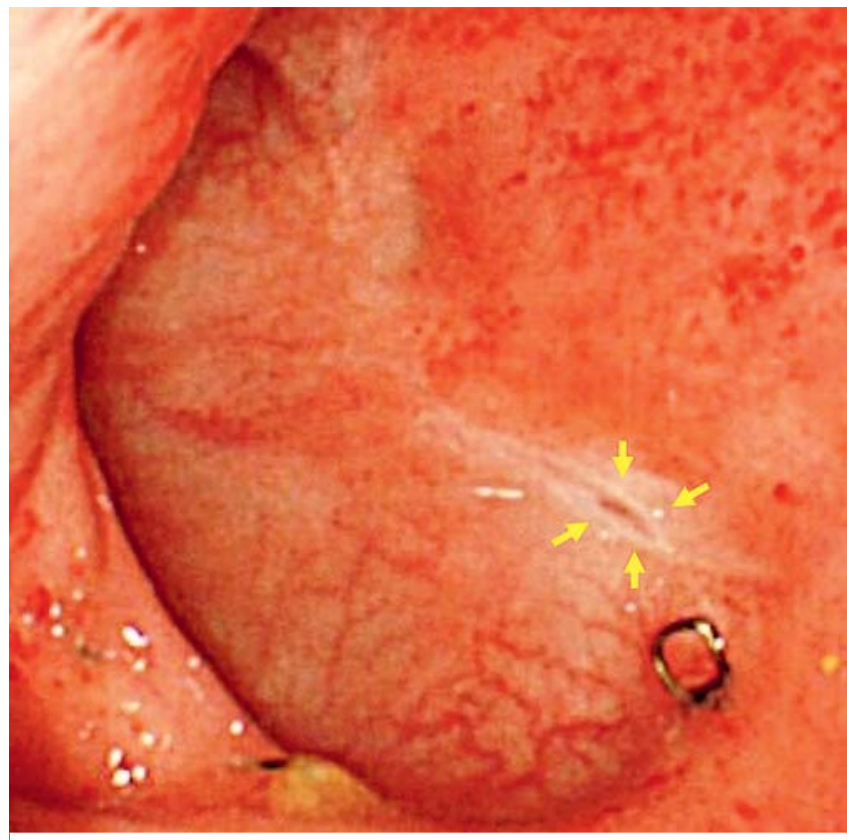

- Fig. 1 Endoscopic examination revealing a small slit-like opening in the anastomotic stoma (arrows).

cessful. However, the endoscopic approach allowed for easy and close visualization of the fistula, and the fistula opening was very small. Because transabdominal surgery is a relatively invasive approach for small fistulas, endoscopy was considered as a viable minimally invasive approach.

In Japan, three distinct endoscopic approaches are used for gastrointestinal leaks and fistulas, namely stent placement, clip closure (TTSC and OTSCs), and injection of tissue sealants. Currently, OTSCs, which are larger and allow for application of greater force, are preferred over TTSCs to close gastrointestinal fistulas. Regarding colon perforation, small perforations ( $<10$ $\mathrm{mm}$ ) can be successfully closed with TTSCs, whereas larger perforation can be successfully closed with OTSCs. However, induration and fibrosis associated with chronic fistulas might result in failure of adequate tissue apposition and consequent inability to satisfactorily deploy OTSCs [6]. In case presented here, the tissue around the RVF was indurate and stretched tightly because of two surgical procedures and the fistula opening was so small that neither anchor nor twin grasper could be applied to close the fistula. Thus, even with OTCSs, closure of RVF was difficult. A large, multicenter, retrospective study of treatment of gastrointestinal defects using OTSCs revealed an overall success rate of $60.2 \%$, but the rate for fistulas (42.9\%) was worse than that for perforations (90\%) and leaks (73.3\%) [7]. Regarding short-term outcomes of OTSCs for recurrent RVFs, the success and healing rates were $26 \%$ and $20 \%$, respectively [3]. As mentioned above, other procedures are necessary to firmly close recurrent RVF. In our case, to achieve a tight closure of the fistula with minimal damage to the tissue surrounding the opening, tiny mucosal holes were made electrically using a needle knife to insert clip jaws on both sides of the fistula opening, which was followed by electrocautery and clip 

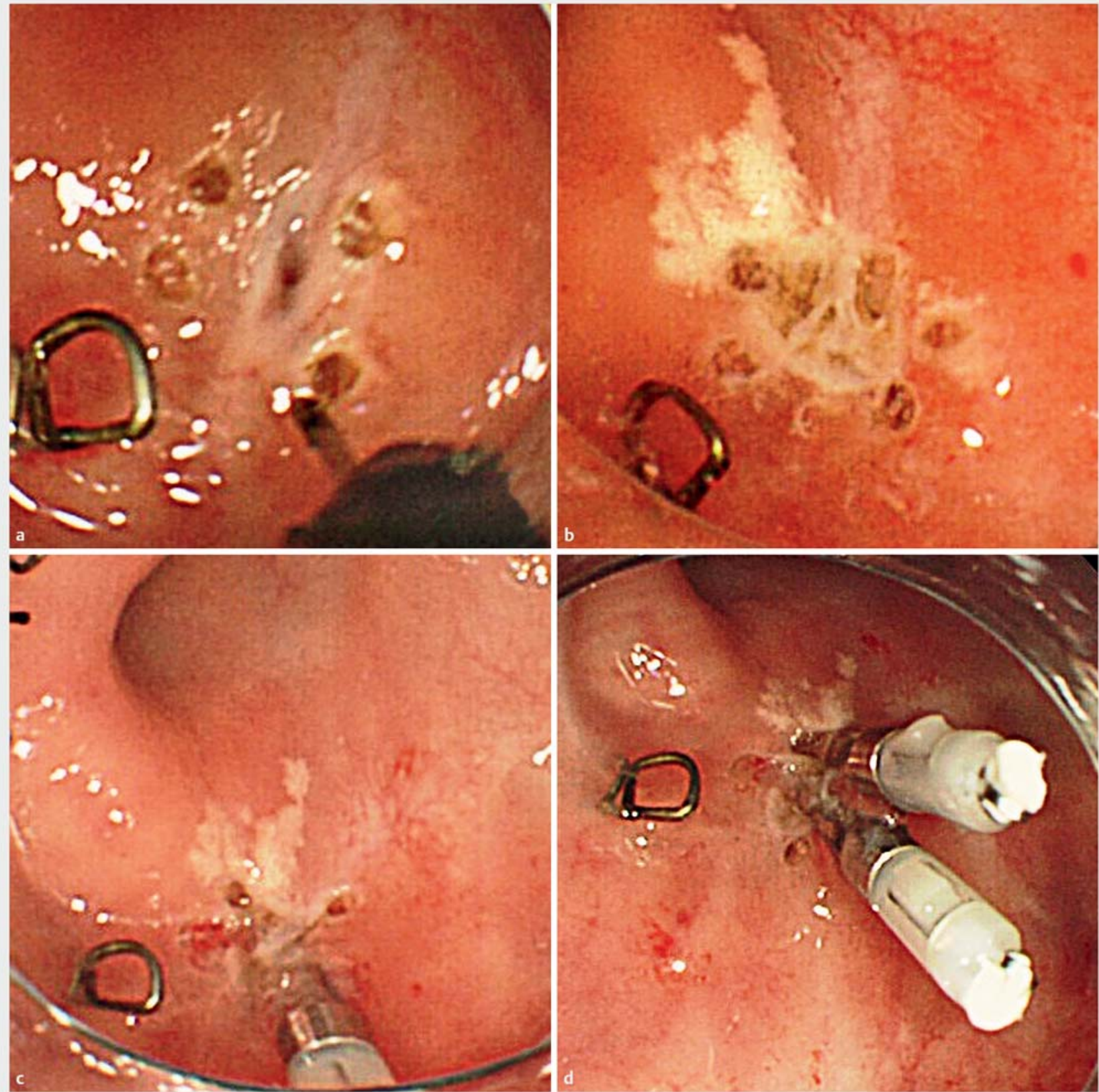

- Fig. 2 Endoscopic procedure. a Four mucosal pin holes on both sides of the fistula are created using a needle knife via electrocautery. b Epithelium around the fistula opening is electrocauterized via argon plasma coagulation. c Clip prongs are inserted in the incisional holes across the opening, which is carefully closed. $\mathbf{d}$ The process is performed twice to achieve complete closure of the fistula.

closure. This procedure was very effective in safe and permanent closure of the fistula without complications. The novel approach of electrocautery and clip closure of gastrointestinal fistulas with pre-procedural endoscopic creation of mucosal pin holes, which has not been previously reported, might have utility for refractory gastrointestinal fistulas with small fistula openings.

The utility of combined endoscopic cautery and clip closure for gastrointestinal fistulas recently has been reported $[8,9]$. This technique, which combines conventional clip application with electrocautery via APC, has been shown to be successful in closure of several types of gastrointestinal fistulas. In an animal model, this approach was suggested to promote fusion of the apposed tissue better than that promoted by a simple closure without electrocautery [10]. Conversely, only one study has reported endoscopic closure of RVF using the Resolution clip [2], which was effective and less invasive but required two applications for permanent closure. Our novel endoscopic approach achieved complete closure of the fistula in a single session. Although simple clip closure without electrocautery may 


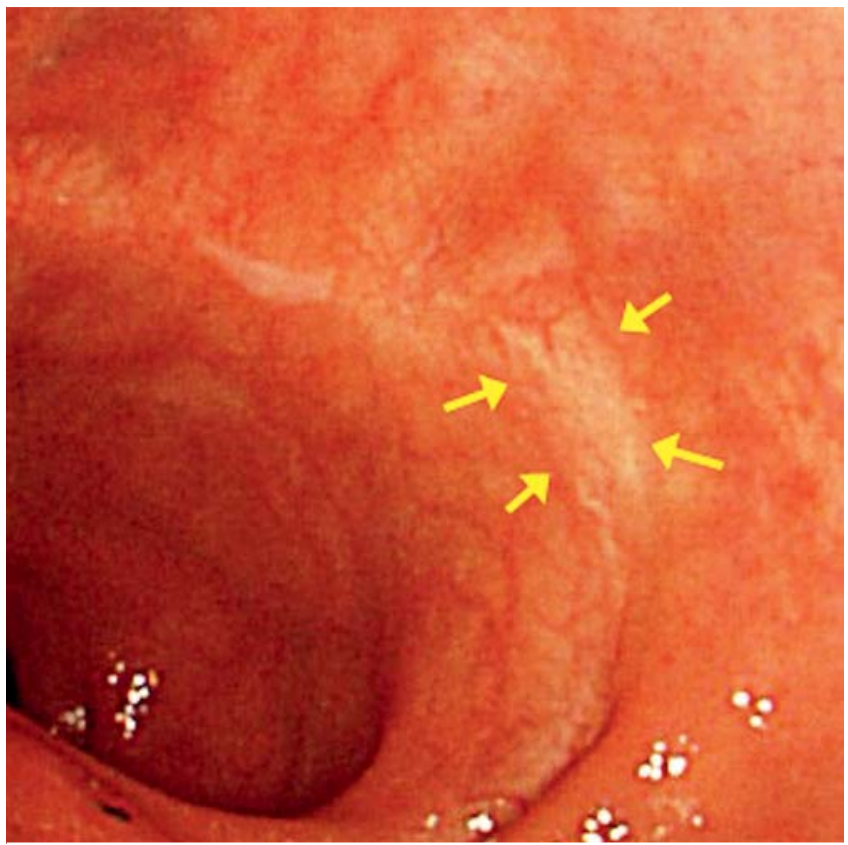

- Fig. 3 Fistula healing is endoscopically confirmed (arrows) at 13 months after the endoscopic procedure.

be sufficient for closure of small gastrointestinal perforations, the combination procedure might be useful for small chronic gastrointestinal fistulas.

\section{Conclusion}

Our novel endoscopic procedure, which included pre-procedural endoscopic creation of mucosal pin holes in combination with electrocautery and endoscopic clipping, was safe, easy, and accessible for refractory post-surgical RVF and should be considered as a less invasive surgical approach for RVF based on the clinical condition of the patient.

\section{Competing interests}

None

\section{References}

[1] Ommer A, Herold A, Berg E et al. German S3-Guideline: rectovaginal fistula. Ger Med Sci 2012; 10: Doc15

[2] John BK, Cortes RA, Feinerman A et al. Successful closure of a rectovaginal fistula by using an endoscopically placed Resolution clip. Gastrointest Endosc 2008; 67: 1192-1195

[3] Prosst RL, Joos AK. Short-term outcomes of a novel endoscopic clipping device for closure of the internal opening in 100 anorectal fistulas. Tech Coloproctol 2016; 20: $753-758$

[4] Vogel JD, Johnson EK, Morris AM et al. Clinical practice guideline for the management of anorectal abscess, fistula-in-ano, and rectovaginal fistula. Dis Colon Rectum 2016; 59: 1117 - 1133

[5] Halverson AL, Hull TL, Fazio VW et al. Repair of recurrent rectovaginal fistulas. Surgery 2001; 130: $753-757$

[6] Banerjee S, Barth BA et al. ASGE Technology Committee. Endoscopic closure devices. Gastrointest Endosc 2012; 76: 244-251

[7] Haito-Chavez Y, Law JK, Kratt T et al. International multicenter experience with an over-the-scope clipping device for endoscopic management of GI defects. Gastrointest Endosc 2014; 80: 610-622

[8] Teitelbaum JE, Gorcey SA, Fox VL. Combined endoscopic cautery and clip closure of chronic gastrocutaneous fistulas. Gastrointest Endosc 2005; 62: $432-435$

[9] Hameed H, Kalim S, Khan Yl. Closure of a nonhealing gastrocutanous fistula using argon plasma coagulation and endoscopic hemoclips. Can J Gastroenterol 2009; 23: 217-219

[10] Felsher J, Farres H, Chand B et al. Mucosal apposition in endoscopic suturing. Gastrointest Endosc 2003; 58: 867-870 